
postcoloniale

\title{
Rabearivelo : "Yo fui negro y vierto la luz »
}

Le manuscrit du Drame de la maison Margon

Xavier Luce

\section{OpenEdition}

\section{Journals}

Édition électronique

URL : http://journals.openedition.org/coma/878

DOI : $10.4000 /$ coma. 878

ISSN : 2275-1742

Éditeur

Institut des textes \& manuscrits modernes (ITEM)

Référence électronique

Xavier Luce, «Rabearivelo : "Yo fui negro y vierto la luz » », Continents manuscrits [En ligne], 9| 2017, mis en ligne le 15 octobre 2017, consulté le 14 novembre 2019. URL : http://journals.openedition.org/ coma/878

Ce document a été généré automatiquement le 14 novembre 2019.

\section{cc) (†) $\odot$}

Continents manuscrits - Génétique des textes littéraires - Afrique, Caraîbe, dispora est mis à disposition selon les termes de la licence Creative Commons Attribution - Pas d'Utilisation Commerciale - Pas de Modification 4.0 International. 


\section{Rabearivelo : "Yo fui negro y vierto la luz »}

Le manuscrit du Drame de la maison Margon

\section{Xavier Luce}

En dehors et au-delà des métiers, des professions

[...] il existe une aristocratie ouverte à tous mais qui n'a jamais été très nombreuse en aucun temps, [...] invisible, dispersée [...], sans diplômes, sans lettres patentes et pourtant plus brillante qu'aucune autre [...]. C'est d'elle que sont sortis les princes les plus véritablement souverains [...], les seuls qui, des années et dans certains cas des siècles après leur mort, dirigent les actions de beaucoup d'hommes. Vous pouvez faire partie de cette aristocratie : [...] la seule condition qu'elle

exige [...], c'est que vous vous soyez livrés immodérément [...] à une certaine forme de plaisir qu'on appelle la Lecture. Valery Larbaud ${ }^{1}$

1 Comprendre Rabearivelo, beaucoup ont cru pouvoir y prétendre. Parmi eux, combien avaient connaissance de son journal d'écrivain? Ce n'est qu'en $2010^{2}$ que les 1833 pages de cahiers d'écolier qu'il appelait ses Calepins bleus devinrent, avec celui de Jean El Mouhoub Amrouche ${ }^{3}$ l'année précédente, le premier «journal écrit directement en langue française par un sujet de l'Empire colonial français » à trouver «son chemin vers la publication $»^{4}$, comme le souligne Almut Seiler-Dietrich dans la revue Présence africaine. Pourquoi ces pages sont-elles restées si longtemps inédites? À cela deux raisons: la première tient à l'investissement financier et au risque éditorial; la seconde, à son contenu : il fallait au moins attendre que les personnes dépeintes, voire incriminées, ne soient plus en mesure de s'en prendre aux ayants droit et à l'éditeur. Ce délai alimenta les spéculations sur la teneur de ces cahiers. Et même sur leur langue d'écriture ${ }^{5}$ : étaient-ils écrits dans la langue maternelle de l'auteur ou dans sa « langue 
adoptive »? La zone de confluence dans laquelle écrivait le poète, à travers l'une et l'autre ${ }^{6}$, entretenait le mystère. Le journal est également traversé par l'existence spectrale de Calepins rouges éminemment sulfureux dont, jusqu'à ce jour, nulle autre trace ne subsiste.

2 À la suite de la publication de ce journal se déploya un important travail de mise en valeur des archives de l'écrivain. C'est par la volonté de la famille, représentée par Brice Rakotomanga, et le concours d'une équipe de recherche composée de chercheurs français et malgaches que des fonds purent être débloqués. Grâce aux moyens financiers et scientifiques de l'ITEM, laboratoire sous les auspices de l'ENS et du CNRS, les archives de celui qui se voulait le premier écrivain moderne de son pays ont pu être inventoriées pour enfin aboutir aux deux tomes de son Euvre complète : un premier contenant, outre les Calepins bleus, une grande partie de sa correspondance et des textes $\mathrm{d}^{\prime}$ ' hygiène morale» propre à son éthique baudelairienne ${ }^{7}$; et un deuxième rassemblant ses recueils de poèmes, ses créations dramatiques et romanesques, ses travaux de traducteur et d'historien ${ }^{8}$. Or, les près de trois mille pages de l'édition papier, qui comprend également un important appareil critique et une présentation génétique pour chacune des œuvres, ne suffisaient pas à contenir l'intégralité des documents retrouvés chez la fille de l'écrivain. C'est ainsi que s'imposa le projet d'une édition numérique, sur la plateforme eman-archives.org ${ }^{9}$. Ce deuxième travail éditorial réalise ce pour quoi Rabearivelo avait lui-même œuvré : la mise en valeur de ses papiers d'écrivain.

Dans les années 1930, on assistait en effet à une première patrimonialisation de la littérature. En 1937, soit l'année de sa mort, un large public pouvait pour la première fois admirer dans le cadre de l'Exposition coloniale des manuscrits autographes d'écrivains célèbres. Ceux-ci étaient agrandis, accrochés au mur, agencés sous vitrine, dans ce qui se présentait comme l'«Ébauche d'un musée de la Littérature ». Cette inauguration «visait à déplacer le centre de gravité du patrimoine littéraire, auparavant assimilé aux œuvres publiées, vers l'activité d'écriture, en rendant visible le travail de l'écrivain, "écriture vive" jusqu'alors invisible ${ }^{10}$ ». À la pointe de l'actualité littéraire et de la vie des idées, Rabearivelo releva cet engouement. La moindre « rognure de Verlaine, celle aussi de Toulet, ne trouve-t-elle pas acquéreur à des prix inouïs?» s'exclamait-il dans ses Calepins bleus ${ }^{11}$. Si bien que, indigène voulant être reconnu comme écrivain national, il était fort conscient, d'une part, de la valeur de ses manuscrits (une littérarité méticuleusement travaillée) et, d'autre part, de la nécessité de constituer un " dossier " à l'intérieur duquel il verserait, tout au long des épisodes marquants de sa vie, des "pièces " (à conviction), cela pour donner à la postérité le matériau nécessaire à l'entretien de sa "légende ». Car pourquoi tenir un journal sinon pour constituer un témoignage de ce que fut sa vie? Tels furent d'ailleurs les deux autres noms de ses Calepins : "Témoins » de ses affres et de ses joies; «Pythagoriques » pour ce qu'ils donneraient accès à la subjectivité d'un sujet de l'Empire et, particulièrement, d'un dandy qui se définissait avant tout «mélanien» (du grec melas, " noir») tout en se pensant comme «latin », pétri de culture française qu'il était. $\mathrm{Si}$, aujourd'hui, seule la mélanine présente un intérêt scientifique - en paléogénétique, notamment -, le terme mélanien était, en 1920-1930, saturé de tout un imaginaire racialisé.

4 Dans cet article, je propose d'éclairer l'aspiration à la fois raciale - identité mélanienne - et nationaliste - aristocrate membre de l'Action française - de Rabearivelo, à partir du 
manuscrit d'une courte nouvelle (16 folios), Le Drame de la maison Margon, mis en lien avec deux autres manuscrits : le brouillon d'une tribune journalistique et celui d'une réponse à une enquête sur la jeunesse noire lancée par l'hebdomadaire Vendémiaire. Bien sûr, ces rapprochements se feront à la lumière des Calepins bleus, sans lesquels il nous serait impossible de trouver une cohérence dans l'œuvre de Rabearivelo, et des documents contenus dans les deux malles conservées par la famille, consultables sur eman-archives.org. À présent que le classement génétique a été établi par Laurence Ink et Christiane Larocca, avec le soutien de l'ITEM, nous pouvons développer sur cette base de travail une étude génétique transversale.

\section{Le contexte de l'écriture : la terre et les morts, de Gobineau à Maurras}

Rabearivelo empruntait à Joseph-Arthur Gobineau, auteur de l'Essai sur les inégalités des races humaines ${ }^{12}$, une terminologie et un concept : il se disait «mélanien », précisait-il, " au sens gobinien ${ }^{13}$ ». Le mot servait à désigner une « race humaine» de peau sombre. Si la prétendue « race » pouvait être répertoriée comme habitante ou originaire des îles austronésiennes ou, plus au sud, de la Tasmanie, au large de l'Australie, elle pouvait également renvoyer à n'importe quel groupe humain jugé barbare. Donc, la revendication de cette catégorie revêt une portée, sur ce plan, comparable à celle de «nègre » ou de "primitif ». L'horizon de Rabearivelo s'apparentait également à celui des félibres. Ce mouvement, d'abord culturel, combattait le centralisme républicain et demandait une autonomie régionale. La figure majeure de cette valorisation du folklore provençal n'est autre que le Prix Nobel de littérature 1904, Frédéric Mistral, dont l'écrivain malgache était un fervent lecteur. Retrouver l'authenticité du terroir et la pérenniser, telle fut leur préoccupation fondamentale. C'est dans ce contexte que s'insère le Provençal Charles Maurras, autre félibre, et son correspondant lorrain Maurice Barrès. Tout au long de sa carrière, ce dernier témoignera de son enthousiasme devant cette conscience régionale dans laquelle, bien évidemment, il s'identifie. En 1930, un ouvrage de luxe des éditions du Cadran rassemble ses divers textes sur la Provence; Rabearivelo l'aura-t-il eu entre les mains? Toujours est-il que son œuvre est imprégnée de cette fibre ethno-nationale. Le 8 juillet 1934, lisant «le plus de livres [qu'il ait] pu retrouver sur Barrès ", il distingue les romans à thèse que constitue le cycle de l'énergie nationale des "œuvres plus discrètes", "plus négligées ", qui ressortent d'une inspiration mystique. C'est bien «la partie théorique qui a trait aux morts et à la terre ${ }^{14} »$ qui retient son attention d'écrivain, précisément, « indigène " mais au sens barrésien ${ }^{15}$. À cela s'ajoute la mythologie personnelle qu'il s'est construite avec Antée : fils de Gaïa, la terre-mère, il fallait à ce géant le contact du sol natal pour se régénérer; pour le tuer, Héraclès dut l'étouffer en le soulevant à bout de bras. Ainsi, chez son Barrès, « il y a là, prolongeant le mistralisme, cet écho, ce reflet, cette image que j'aime et qui sont ceux de la geste d'Antée, seul symbole de la force et de la noblesse spirituelles ${ }^{16}$ ». Telle est la posture de l'écrivain indigène que s'est forgée Rabearivelo. Bien sûr, cet imaginaire de l'ethno-nationalisme, "la terre et les morts ", s'est nourri de la pensée de Charles Maurras. Ces trois figures de l'identité culturelle française forment donc un tronc commun à partir duquel on peut tenter de cerner l'itinéraire de l'écrivain malgache. 
6 À mesure que l'Empire parachève ses guerres de conquête, cette mouvance littéraire est réinvestie par des sujets de l'Empire ${ }^{17}$. On relève, en effet, une transposition sur le terrain de la géographie par le biais d'un réseau d'analogies entre Métropole et Colonie. Exemple notoire, la colline royale d'Ambohimanga, qu'Européens et Malgaches dits " évolués " s'accordent à célébrer comme une autre colline inspirée, renvoie à cette colline de Sion à la frontière des Vosges et de la Meurthe-et-Moselle qu'invoque Maurice Barrès. Un « lieu de mémoire ", porteur d'un sentiment national, se transfuse de l'occupant à l'occupé dans une consanguinité littéraire et esthétique. Rabearivelo en parle comme d'une "adhésion morale » mais, dans son esprit, cela correspond bien à un pacte de sang : «Ai donné mon adhésion à Maurras et à Daudet - la main dans celle du poëte Lebrau - au nom de l'Esprit (qui ignore ou dédaigne la Lettre) et du Sang (qui se moque de la Couleur) ${ }^{18} »$. Expression baroque et lourde de symboles qui montre bien comment la portée d'un texte littéraire peut être renégociée en colonie. Prestige des lettres, oui, mais encore "noblesse spirituelle ». Sur ces références étrangères viennent se greffer d'autres références, celles-là malgaches et, plus spécifiquement, merina et hova. Notions ô combien délicates, source de divisions artificielles qu'instrumentalisèrent, à partir de 1828 , une oligarchie naissante puis les autorités coloniales à travers une "politique des $\operatorname{races}^{19}$ ". Aux yeux du chef de district qu'accompagnait Rabearivelo pour lui servir d'interprète, il était admis que "Madagascar n'est pas une unité ethnographique », que « les diverses tribus malgaches peuvent se ranger en trois grandes races ». D'un côté, un élément africain, de l'autre un élément non africain: malais; à quoi s'ajoutait un métissage arabe. Grammairien, connaissant l'existence de la famille des langues austronésiennes, l'administrateur Lucien Montagné était tout aussi certain qu'il y avait "d'abord les Hovas », étant donné qu'ils « proviennent de Malaisie ${ }^{20}$ » et ensuite les autres ethnies. Et, en effet, ce sont bien les populations étiquetées Merinas ou Hovas qui ont le plus marqué les esprits européens. Par le lamba, habit traditionnel, qu'ils portent comme les Grecs et les Romains la toge, ils exerçaient une attraction chez les Européens. Ce sont eux qui occuperont la majorité des postes de fonctionnaires, comme l'indique le rapport Pégourier de 1927-1928. Ceci montre le rôle symbolique que jouait le sang, puisqu'il était question d'ethnies plus ou moins étanches et aux contours délimités.

\section{Le Drame de la maison Margon}

$7 \quad$ Parmi les textes non retenus pour les deux tomes de l'édition papier, faute de place, il y a cette nouvelle, Le Drame de la maison Margon. Le manuscrit est en bon état, lisible, et porte la date du 9 février 1925. Il s'agit d'un cahier d'écolier de cinquante pages dont dix-sept feuillets sont rédigés. Le récit nous offre un tableau de mœurs dans le Tananarive des années 1920: un ménage de colons en ruine, la femme mondaine et lascive, son mari fruste au point de devenir proxénète ; leur boy, délicat et l'esprit vif, prolongeant vaille que vaille le luxe de la maison. Tel est le tableau que nous rapporte le narrateur, d'après le récit que lui en fit ce même boto. Le drame, en effet, s'enchâsse dans un autre récit, celui de deux jeunes Malgaches bilingues qui se retrouvent dans les rues de Tananarive pour se raconter: l'un, ses mésaventures (comment il fut déporté au large des Comores) et sa vie de boy (comment, à son retour d'exil, il en fut réduit à se louer); l'autre, ses "nombreux acquis intellectuels ${ }^{21}$ ". C'est à ces échanges que nous devons le récit du Drame de la maison Margon. Le narrateur se souvient comment, alors 
qu'il venait d'entrer au service du Cercle de Tananarive comme bibliothécaire, « [il fit] la connaissance d'un garçon extraordinaire, originaire du pays betsileo, qui s'appelait Barthélémy Randevo ». Il tente de se souvenir, parmi les histoires qu'il lui a contées - si nombreuses, que s'il devait toutes les « consigner", celles-ci « rempliraient autant de volumes que la Comédie humaine »-, de sa première expérience d' « homme à tout faire » au sein d'une maison de passe. Il est significatif que cet effort de mémoire de la part du narrateur s'observe, sur le manuscrit, à travers une recherche de style: deux propositions se chevauchent, deux métaphores interfèrent. L'auteur tente de trouver le ton juste pour conférer une certaine sensualité à son personnage. Il songe d'abord au métier à tisser, à la trame d'une étoffe ou d'un tissu ; puis, finalement, à une pelote. Pourquoi tant d'attention? C'est que tout l'enjeu de la nouvelle réside dans la capacité de Barthélémy Randevo à transcender sa vie de boy au moyen du Langage et de l'Esprit.

Figure 1. J.-J. Rabearivelo, Le Drame de la maison Margon, $f^{\circ} 2 \mathrm{r}$

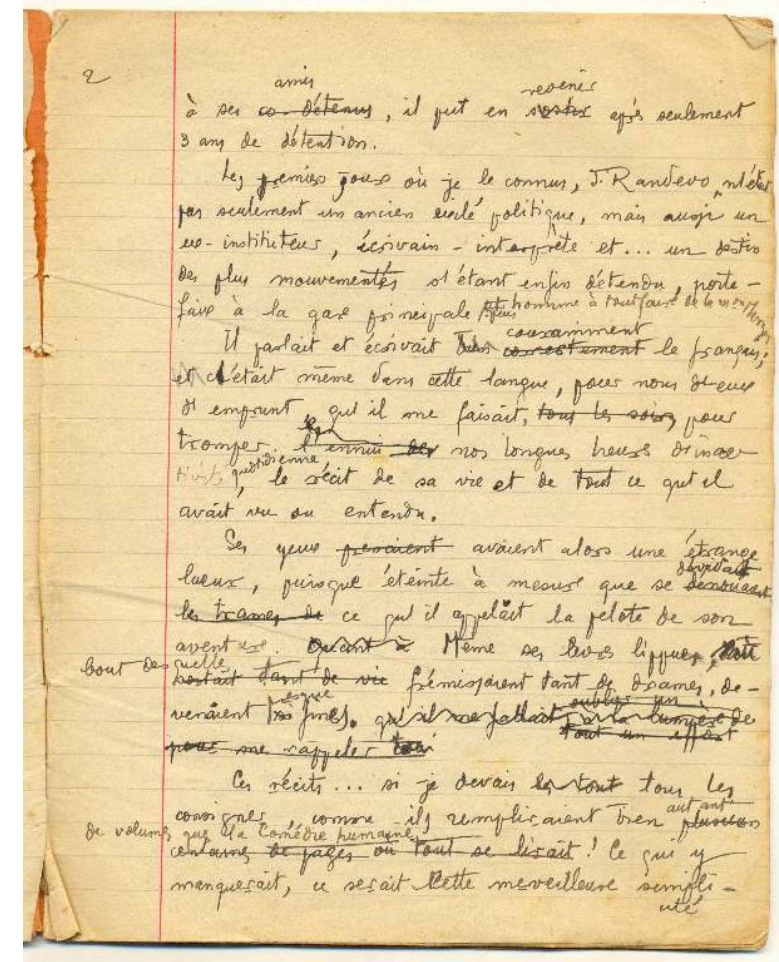

Droits : famille Rabearivelo

\section{Transcription du document}

Ses yeux avaient alors une étrange lueur, presque éteinte à mesure que se [dénouaient] dévidait [les trames de] ce qu'il appelait la pelote de son aventure. [Quant à] Même ses lèvres lippues, [d'où sortaient] au bout desquelles [tant de vie] frémissaient tant de drames, devenaient [si] presque fines. Qu'il me fallait à la lumière de tout un effort oublier un pour me rappeler tou $\pi$

Ces récits... si je devais [les tout] tous les consigner, comme ils rempliraient bien [plusieurs centaines de pages où tout se lirait] autant de volumes que la Comédie humaine! Ce qui y manquerait, ce serait cette merveilleuse simplicité| |ा

8 Le pouvoir de la langue est tel que ses «lèvres lippues» deviennent "presque fines ». Comme si le portrait moral (un écrivain-interprète et enseignant) l'emportait sur son 
apparence physique (un jeune Betsileo) de telle sorte que son Esprit estompe ses traits " négroïdes ». Cette distorsion du personnage, entre ses qualités intellectuelles et son faciès, provient évidemment de la théorie de l'inégalité des races : des lèvres, un nez épatés, le prognathisme, etc., autant de particularités sur lesquelles on s'évertua à fonder une science des caractères et des pulsions ataviques. L'anthropométrie, introduite à Madagascar au début du $\mathrm{xx}^{\mathrm{e}}$ siècle, participe au «renforcement des barrières catégorielles et raciales propres au régime des colonies ${ }^{22} »$. Ce développement n'est pas étranger non plus à l'image dépréciée que se fait Rabearivelo du Nègre tel qu'il ressort de ces mesures et des théories puisées chez des penseurs racialistes. Ainsi, dans les années 1930, il se rend dans un asile et accorde une interview à un membre de son entourage par ailleurs directeur du journal dans lequel celle-ci sera publiée ${ }^{23}$ :

Camille de Rauville m'a envoyé hier soir le projet, le topo, qu'il avait tiré de l'interview qu'entre deux whiskies je lui avais accordée, à mon retour de chez les fous, dimanche soir, chez Fumar.

[...] J'ai intitulé l'interview "Au pays de Lombroso ». M'a beaucoup servi le beau livre du $\mathrm{D}^{\mathrm{r}}$ Vinchon ${ }^{24}$ que déjà, il m'en souvient, j'avais utilisé il y a quelques dix ans dans un article sur l'art et la folie.

Reviendrai plus amplement, une autre fois, sur les visions inoubliables que j'eus, làbas, dans l'enceinte même de l'asile, et dans la nuit de samedi et dans la journée de dimanche.

Des visions tragiques et, tout ensemble, grotesques, la Misère elle-même ${ }^{25}$.

Cesare Lombroso et Gobineau font partie de la bibliothèque mentale de Rabearivelo et constituent, dans son œuvre, un substrat sensible à maints endroits. En outre, pour évoquer le frémissement produit par les mots sortant de la bouche de Randevo, il aura d'abord écrit «si fines». Son personnage s'apparente à l'archétype du «nègre romantique " ou du "noble primitif » chez qui les traits physiques se dénégrifient à mesure que sa personnalité singulière émerge et l'extrait de sa Race. Au vu de ce premier jet, Randevo semble comme être touché par la grâce : il jouit de qualités oratoires et d'une élégance dans l'expression.

Pourtant, adopter le point de vue d'un boy reste un parti pris fort. Le choix de l'ériger en source privilégiée de l'histoire (Le Drame de la maison Margon) et de l'Histoire (Tananarive sous l'occupation française) constitue en effet un déplacement épistémologique de taille. Le rapport de force narratif est renversé: le regardé (l'indigène) devient le regardant (le colon). La nouvelle tend à s'inscrire en porte-à-faux de la littérature coloniale, ne serait-ce que par l'emploi de l'expression "pays betsileo » plutôt que race betsileo, qui fait du personnage moins un type qu'un individu doué d'une personnalité et, comme on le verra, de tourments psychologiques. De fait, si Barthélémy Randevo en est à réduit à cette situation en deçà de ses compétences d'instituteur et d'écrivain-interprète, c'est bien moins en raison d'une appartenance raciale fixée telle par l'Administration et, en dernière instance, par la «bibliothèque coloniale ", qu'en raison de son statut légal d'indigène. Le terme betsileo redevient un marqueur géographique et indique l'éloignement familial de Barthélémy Randevo et, a fortiori, son origine rurale. Car le royaume betsileo se trouve dans la partie sud des hauts plateaux de l'Imerina. Il est le fruit de "populations régionales" qui se sont, à travers les siècles (entre le $\mathrm{XV}^{\mathrm{e}}$ et le $\mathrm{XVIII}^{\mathrm{e}}$ siècle, note Manassé Esoavelomandroso ${ }^{26}$ ), individualisées au sein d'un espace politique autonome en relation avec d'autres populations de régions limitrophes telles que, dans l'Ouest, les Mahafale, Sakalava, dans le Centre, les Merina, Bara, etc. Ainsi, de ce brillant Betsileo déclassé, Rabearivelo en 
fait sur le plan politique le représentant d'un "arrière-pays culturel ${ }^{27}$ » - tel que le définit l'intellectuel caribéen Edouard Glissant dans Le discours antillais.

11 Sur un plan littéraire, le personnage s'incarne comme un "conteur primitif ${ }^{28}$ ». Il s'oppose en cela à ce jeune bibliothécaire imbu de littératures européennes sous lequel l'on décèle, en creux, un autoportrait de l'auteur : Rabearivelo avait lui-même été aidebibliothécaire au Cercle de l'Union à Tananarive où il « dévore littéralement le fonds ${ }^{29}$ !", note Serge Meitinger. Déjà se dessine le portrait du mélanien latinisé ou " naturellement latin chez les Mélaniens. Et avec les traits de ceux-ci ${ }^{30} »$, comme il semble vouloir en rire dans ses Calepins bleus. Pour avoir trop étudié son sujet ( les Européens »), à la fois bibliovore et ethnologue à rebours ${ }^{31}$, il jouirait d'une surconscience culturelle : ayant accès à ce que les Européens discernent mal (la subjectivité des indigènes) et observant ces mêmes Européens sous toutes leurs coutures (le boy ayant accès à leur intimité et à leurs livres).

Le portrait moral de Barthélémy Randevo fonctionne en contrepoint de celui du personnage narrateur, l'un et l'autre se constituant comme une fable ontologique dans laquelle se regarde Rabearivelo. Ce thème de l'acculturation et de l'authenticité est chez lui un thème majeur. L'Interférence, titre d'un de ses romans, c'est l'éblouissement qu'engendre la rencontre de lumières contraires: la source originelle et la source choisie à l'étranger, explique Serge Meitinger. C'est une métaphore du "contact» imposé par l'Europe aux cultures étrangères, ou de l'impossible contact, comme le suggère le roman et comme l'affirmera avec aplomb Aimé Césaire dans son Discours sur le colonialisme. Dans cette optique, la dualité que les personnages composent (d'un côté celui « tout de culture livresque », de l'autre le « conteur primitif ») devient le symbole d'une philosophie de l'Histoire. Aux yeux de Rabearivelo, l'expansion européenne sera victime de son "génie "; le Nègre réfléchira la lumière blanche. Anticipation similaire que l'académicien Paul Valéry, à propos du péril jaune, exprime dans une veine technocratique et autoritaire ${ }^{32}$. Or, la lecture de ses deux lettres sur la Crise de l'esprit ${ }^{33}$, la fréquentation de ses poèmes, mais aussi d'autres textes, notamment ceux rassemblés dans Regards sur le monde actuel ${ }^{34}$, particulièrement les pages ayant trait à l'Orient, ont beaucoup marqué l'imaginaire de Rabearivelo. Le déclin de l'Occident, miné par son désintéressement pour les choses de l'Esprit ou, au contraire, par son agitation frénétique, soutient Paul Valéry, inscrit le continent européen dans la longue suite des âges qui ont vu « disparaître » des civilisations les unes plus brillantes que les autres; car toutes sont «mortelles ». C'est pourquoi, au lendemain de la Grande Guerre, il tient à tirer les conclusions de cette barbarie survenue cette fois au plein cœur de l'Europe. Ce spectre l'accompagnera jusqu'à sa mort, en 1945.

Rabearivelo hésite sur le prénom à donner à son héros, d'abord Jacques, Barthélémy puis Randevo : $\mathrm{Ra}+$ andevo, $r a$ étant un préfixe (tel $\mathrm{Ra}+$ bearivelo), andevo signifiant « esclave » en malgache. L'onomastique nous renseigne sur l'intention de l'auteur. Il est bien remarquable qu'il choisisse comme personnage principal un «fils d'esclave », lui, Rabearivelo, fier du sang royal hérité par sa mère (la lignée déchue des Zanadralambo), qui ailleurs indexe les gens en fonction de leur origine servile ${ }^{35}$. Ce primat accordé au domaine spirituel (ignorant la Race, sublimant le phénotype), rejoint l'angoisse formulée par Paul Valéry au lendemain de la Grande Guerre sur l'avenir et la politique de l'esprit. Reste que ce portrait moral d'un jeune exilé originaire du pays betsileo n'est pas anodin et, au regard de la vision politique de Rabearivelo (élitisme de Race et culte 
des Lettres), occupe une place importante. Un deuxième document exhumé des archives familiales nous permet de prolonger cette analyse.

\section{Réponse de Rabearivelo à une enquête de Vendémiaire}

Que l'Europe fût «moralement, spirituellement indéfendable ${ }^{36}$ " n'était pas la préoccupation majeure de Rabearivelo. Moins pamphlétaire, subversif tout de même, et surtout plus prudent, il y voit essentiellement une occasion de prendre voix au chapitre. Il incarne une posture de "mélanien" ou de "nègre", en tous les cas d'intellectuel colonisé. Ce faisant, il s'inscrit dans les traces de ses illustres devanciers; au premier chef, René Maran, et, bien sûr, Rabindranath Tagore.

C'est ainsi que l'enquête menée par l'hebdomadaire parisien Vendémiaire sur la jeunesse noire lui donne l'occasion de "secouer les cendres", confie-t-il dans son journal à propos d'un tout autre sujet, de «ma FINE CIGARETTE». Tel est ce à quoi il aspire : n'avoir de compte à rendre à personne, à l'abri du besoin, et pouvoir juger des «Livres » et des « Hommes» depuis chez lui ${ }^{37}$. C'est dans cette perspective qu'il choisit de répondre à ce questionnaire de Vendémiaire ${ }^{38}$.

Figure 2. J.-J. Rabearivelo, « Réponse à une enquête de Vendémiaire »

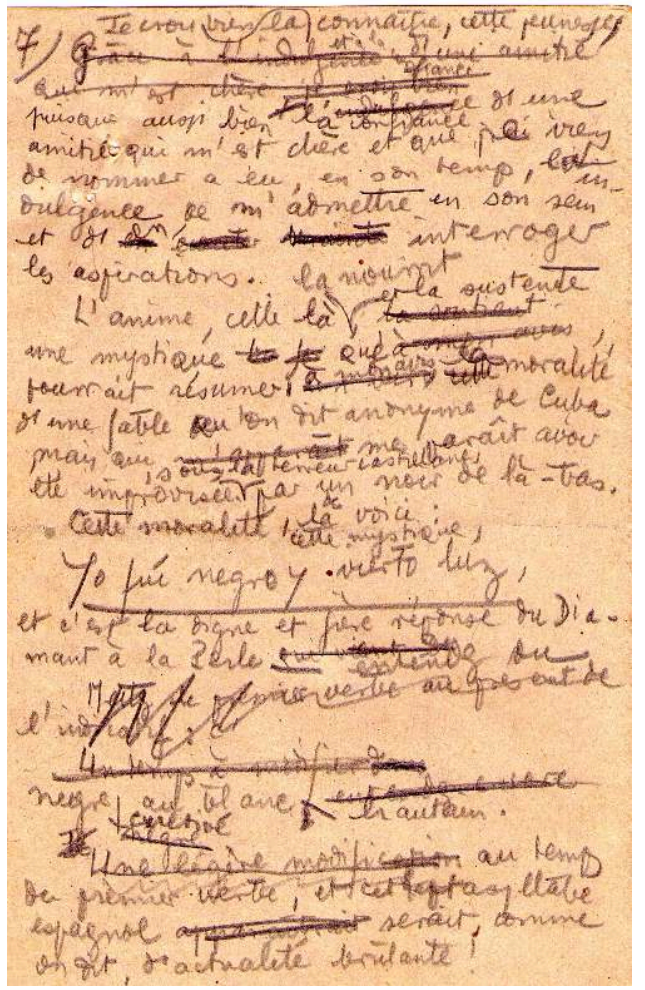

Droits : famille Rabearivelo

Il a recours à une métaphore pour formuler un discours sur le sens de l'Histoire. Il s'appuie sur «la moralité d'une fable qu'on dit anonyme de Cuba» dans laquelle le Diamant, qui est "issu du charbon " précise-t-il, représente le mélanien; tandis que «la Perle (fille de la mer) », représentant bien sûr le Latin ou la «race blanche », 
appelle un mouvement de l'Histoire qui s'annonce inéluctable. Ainsi, cette fable se donne à lire comme une prémonition du siècle au bout duquel s'entrevoit un « temps postcolonial », post au sens de ce qui viendrait après changer l'équilibre mondial. Voici ce que dit la Perle au Diamant : «Je vaux beaucoup plus que toi / Tu es né du charbon noir et moi de la mer bleue ", à quoi il répond: « Tu n'as aucun mérite / Tu as toujours été et resteras blanche / Moi j'étais noir et suis devenu lumière »: "Yo fui negro [y] vierto la luz. » Et Rabearivelo de conclure par cette subversion de la célèbre phrase de Paul Valéry: "Il y aura toujours de par le monde des hommes qui, ayant appris l'Histoire et sachant que nulle civilisation n'est immortelle, ne s'étonneront de rien même de l'intensité de la lumière versée par le Nègre... »

Rabearivelo affirme avec beaucoup d'audace, dans les colonnes d'un organe de presse français, au milieu d'écrivains coloniaux (Marius-Ary Leblond, Pierre Mille, André Demaison et René Maran $^{39}$ ), une fierté de race qui dépasse le cadre de l'île, mais interpelle les femmes et les hommes noirs de génie où qu'ils se trouvent. La majuscule au mot nègre renvoie bel et bien à la catégorie de colonisé à laquelle il s'identifie, dans le champ littéraire français (dans la lignée de René Maran), et qu'il met en fiction à travers le portrait d'un « boy ».

Il était nécessaire de mettre en perspective Le Drame de la maison Margon avec un texte postérieur d'une dizaine d'années afin de montrer comment, dans cette fiction, émerge une conscience politique singulière. Il faut à présent l'éclairer à la lumière d'un manuscrit qui semble annoncer, un mois auparavant, les tribulations de Barthélémy Randevo. Il s'agit du brouillon d'un article de presse.

\section{Une tribune dans la presse}

En mettant en relation ces deux manuscrits, le brouillon d'une tribune et celui d'une nouvelle, l'étude génétique met en valeur leur double thématique commune: les mouvements nationalistes d'une part, et de l'autre le capital symbolique dont jouit le français ${ }^{40}$ au sein de la «phalange Rabearivelo ».

Ces deux textes sont en effet contemporains d'un évènement majeur dans l'histoire du nationalisme malgache, connu à Madagascar sous les initiales de V.V.S. : Vy Vato Sakelika (Fer, Pierre, Ramification). Mouvement pacifiste et intellectuel, la "société prétendue secrète " ou " quasi secrète », corrige Rabearivelo, réunit des étudiants de l'école de médecine, des instituteurs, des pasteurs, des journalistes et des hommes de lettres. Les luttes politiques furent animées par Jean Ralaimongo et c'est le pasteur Ravelojaona qui en donnera l'impulsion à travers une série d'articles publiés de 1913 à 1915 dans la revue Ny Mpanolo-tsaina au sein desquels le Japon, autre puissance coloniale, est perçu comme un modèle de société. L'Empire japonais, de son côté, élaborait « un État moderne en se référant constamment à des modèles occidentaux ${ }^{41}$ ». L'occidentalisation nippone, par la conservation de sa langue et de sa culture, tend à ces nationalistes malgaches un miroir où ils appréhendent l'avenir de la grande île. Toute association « indigène " étant proscrite, les premières arrestations surviennent en décembre 1915. Après la prison s'ouvre un « procès » qui les condamne à l'exil. Puis, une fois la situation en métropole rétablie, l'amnistie est officiellement prononcée le 22 novembre 1922. L'année suivante, les prisonniers politiques de la V.V.S. font leur retour. Tananarive connait alors une petite effervescence culturelle: des revues se créent, les échanges se font plus libres, une génération urbaine et lettrée se forme. C'est 
ici qu'intervient Rabearivelo; consultons d'abord le brouillon du Drame de la maison $\operatorname{Margon}^{42}$, puis un projet manuscrit de tribune à paraître ${ }^{43}$.

Figure 3. J.-J. Rabearivelo, Le Drame de la maison Margon, $\mathrm{f}^{\circ} \mathrm{rr}$

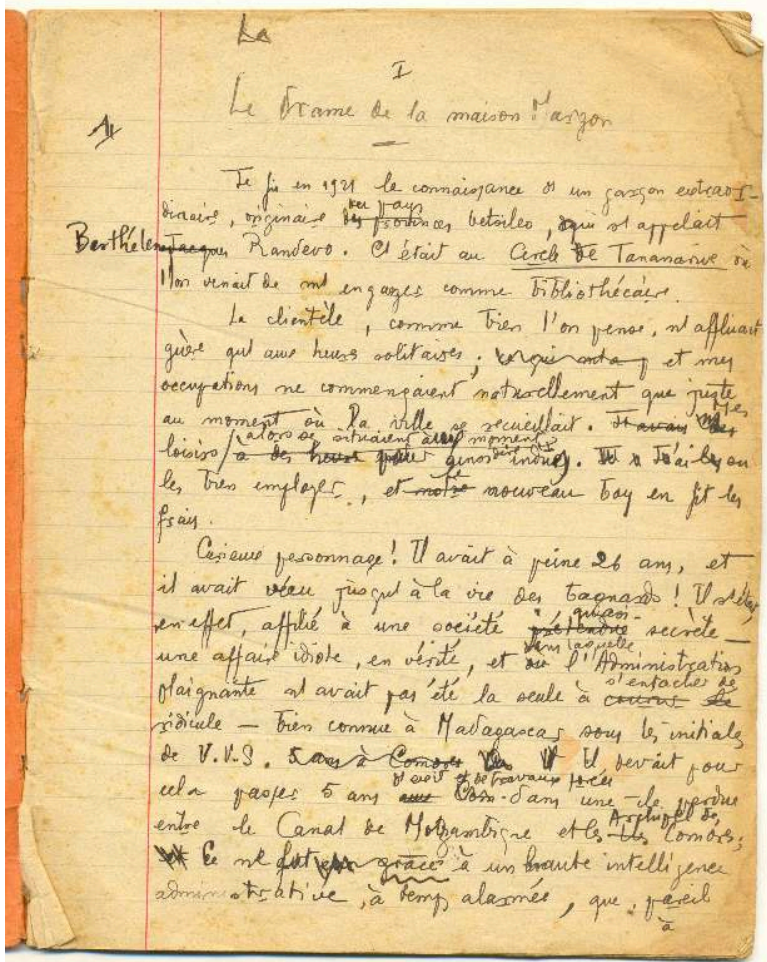

Droits : famille Rabearivelo

\section{Transcription du document}

Je fis en 1921 la connaissance d'un garçon extraordinaire, originaire du pays betsileo, qui s'appelait Barthélemy Randevo. C'était au Cercle de Tananarive où l'on venait de m'engager comme bibliothécaire. भा

La clientèle, comme bien l'on pense, n'affluait guère qu'aux heures solitaires; et mes occupations ne commençaient naturellement que juste au moment où la ville se recueillait. J'ai su les bien employer, et le nouveau boy en fit les frais. बा

Curieux personnage ! Il avait à peine 26 ans, et il avait vécu jusqu'à la vie des bagnards! Il s'était en effet, affilié à une société quasi secrète - une affaire idiote, en vérité, et sur laquelle l'Administration n'avait pas été la seule à s'entacher de ridicule - bien connue à Madagascar sous les initiales de V.V.S. Il devait pour cela passer 5 ans d'exil et de travaux forcés dans une île perdue entre le Canal de Mozambique et 1'archipel des Comores. ๆ 
Figure 4. J.-J. Rabearivelo, « Non une amnistie, mais une révision !», projet de tribune

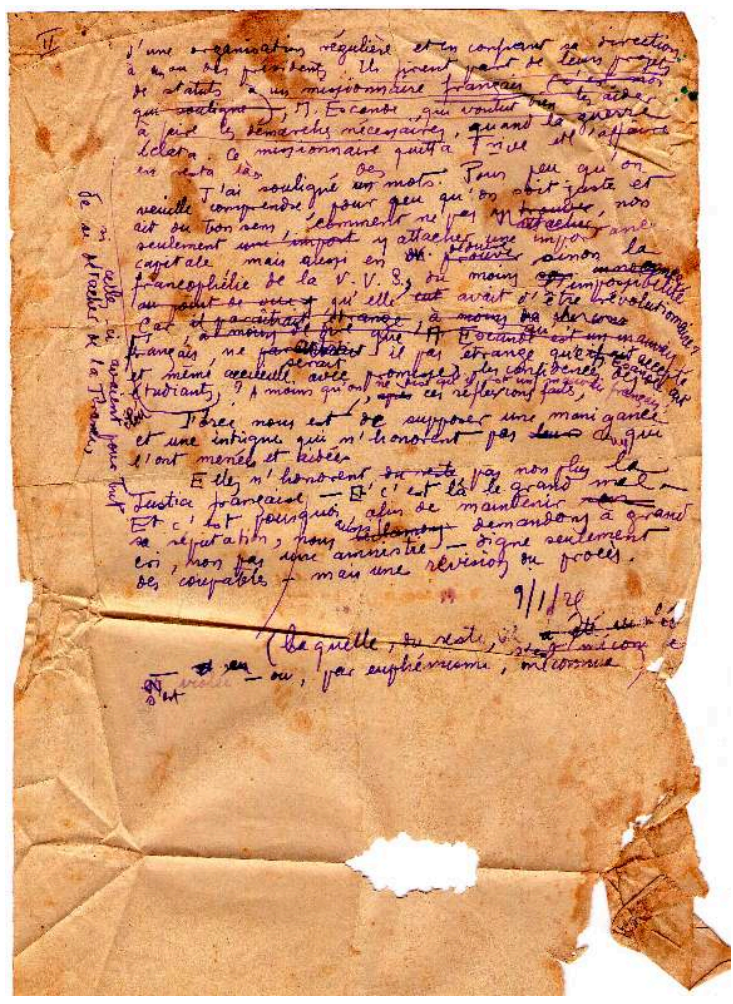

Droits : famille Rabearivelo

21 Dans ce feuillet manuscrit daté du 9 janvier 1925, soit exactement un mois avant le brouillon du Drame de la maison Margon, nous trouvons trace de l'engagement de Rabearivelo : il s'indigne du traitement arbitraire réservé à ses aînés. Il fait valoir l'idéal humaniste que prétend incarner la «Justice française » et écrit : « Afin de maintenir sa réputation, nous demandons à grand cri, non pas une amnistie - digne seulement des coupables - mais une révision du procès ${ }^{44} »$. Cette intransigeance juridique que déploie le jeune poète ne vaut qu'en fonction d'un attachement sentimental à la Mère-Patrie (on note cependant une légère ironie) dont il a découvert les Muses alors qu'il n'avait " pas vingt ans $^{45}$ ». Il s'attache à souligner la «francophilie ${ }^{46}$ » de ses compatriotes injustement incriminés. Déjà à cette époque, Rabearivelo était régionaliste et plaidait pour une culture "franco-malgache ». Sa vision de l'avenir de Madagascar tient dans cette phrase des Calepins bleus: «Une langue encore pure, celle de la tribu, qui ne sera jamais plus retrouvée mais que les Poëtes auront pour mission de mallarmiser, pour peu qu'ils aient le désir de survivre ${ }^{47}$ ". À ses yeux, c'est au prisme de la langue et, à travers elle, de la rencontre entre les cultures, que doivent s'envisager les rapports de Madagascar à la métropole. Mallarmiser la langue malagasy, donc. Pour ce faire, il convient au préalable de s'approprier le "génie de la langue » française, en percer les " $\operatorname{arcanes}^{48}$ ». Rabearivelo, d'abord secrétaire d'un chef de district de 1916 à 1919, puis correcteur d'épreuves à La Tribune de Madagascar, attache un soin extrême à la grammaire et au choix des mots. Rabearivelo fétichise la langue de Ronsard.

De cette obsession du bien parler, nous trouvons trace dans le manuscrit de la Maison Margon. Le portrait moral du personnage de Barthélémy Randevo dessiné au seuil du récit est révélateur. Désirant louer les compétences linguistiques de son héros, il écrit d'abord que Barthélémy Randevo "parlait et écrivait très correctement ». Puis, 
s'avisant du ton professoral malencontreux, rature et remplace par « couramment ». Ce n'est pas une simple correction puisqu'aussi bien c'est tout l'enjeu du paragraphe. Les deux compères partagent un talent précieux qui, on le devine en creux, dénote un prestige non accessible au commun des Malgaches, l'enseignement étant dans une situation déplorable. Se mallarmiser devient à la fois le maître mot d'une attitude à avoir par rapport à l'acquisition de connaissances, ainsi qu'il le développera dans la revue $\mathrm{Ny}$ Fandrosoam-baova ${ }^{49}$, et d'une manière d'être au monde. Il théorise et développe un discours sur la stratégie à adopter pour se prémunir contre la dilution culturelle. Il est avant tout un nationaliste hova, dans le sens où il porte la nostalgie du royaume consolidé par Radama ${ }^{\text {er }}$ dans la première moitié du xix siècle. Pour Rabearivelo, écrivain national hova, français par adoption et par stratégie, Radama ${ }^{\mathrm{er}}$ portait un projet politique et littéraire: s'opposer à la francisation intégrale au nom de sa «Personnalité » et du « fonds poétique ${ }^{50}$ » de la Race, tout en s'imprégnant de la culture européenne. Le contre-exemple fourni par l'ethnologue Victor Segalen donne matière à réfléchir. Dans son roman de $1907^{51}$, ce dernier mettait en scène la déliquescence d'une société polynésienne depuis que des vaisseaux britanniques y accostèrent avec bibles et alcools, fusils et concupiscence. Le point d'orgue de cette fiction est l'oubli, le moment où le Conteur, gardien de la mémoire, omet un chaînon au récit des origines. La communauté décrite par Segalen devient alors «sans passé ». Tel est le spectre contre lequel écrit Rabearivelo, le fil rouge de son œuvre: ne pas devenir des "Immémoriaux» ou des "Impersonnels ${ }^{52}$ ». Le risque inverse étant l'acculturation. C'est ainsi qu'apparait, au plus proche du «cœur végétal » et en phase avec «l'âme éternelle» de Iarive, Barthélémy Randevo. Son naturel, constate amèrement le narrateur, est ce

Figure 5. J.-J. Rabearivelo, Le Drame de la maison Margon, $\mathrm{f}^{\circ} 3 \mathrm{r}$

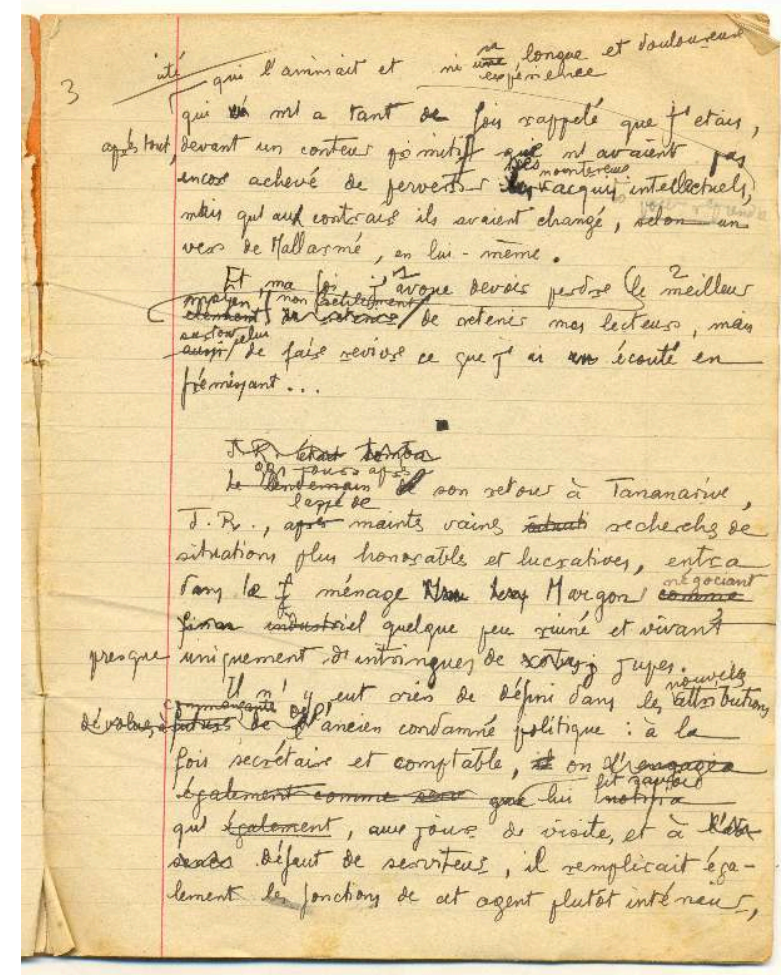

Droits : famille Rabearivelo 


\section{Transcription du document}

[...] qui m'a tant de fois rappelé que j'étais, après tout, devant un conteur primitif que n'avaient pas encore achevé de pervertir les nombreux intellectuels ni [une] sa longue et douloureuse expérience, mais qu'au contraire ils avaient changé, selon un vers de Mallarmé, en lui-même. बा

Toujours l'obsession mallarméenne de parvenir à cette osmose du langage. Le vers que Rabearivelo a en tête est celui du premier quatrain du « Tombeau d'Edgar Poe »: «Tel qu'en Lui-même enfin l'éternité le change. » C'est que le sonnet de Mallarmé l'avait particulièrement frappé. Il y reviendra quelques années plus tard, dans un poème où sera d'entrée mis en exergue (scrupuleusement souligné comme à son habitude, en signe d'hommage érudit) ce fameux vers de Mallarmé. L'alexandrin se charge alors, sous la plume de Rabearivelo, d'un sens bien plus crucial compte tenu du contexte d'énonciation.

Donner un sens plus pur aux mots de la tribu

et l'imprégner du sang de mes morts que nos combes

ombreuses et nos monts ensoleillés ont bu :

mission périlleuse et double qui m'incombe!

Qui donc me donnera de pouvoir fiancer

l'esprit de mes aïeux à ma langue adoptive,

et mon cœur naturel, calme et fier au penser

pervers et sombre de l'Europe maladive,

pour susciter des chants où ma pure entité

se précise selon le rythme et la cadence

de l'Intuition, et de toute influence

s'affranchit, changée ainsi par l'éternité ${ }^{53}$ ?

L'alexandrin de Mallarmé offre à l'oreille du poète malgache une caisse de résonnance où s'entend, dès lors, sa détresse, en tant qu'intellectuel porteur d'un « sentiment national ». La « mission périlleuse » de « donner un sens plus pur aux mots de la tribu » fait écho à l'étiolement de celle-ci, pour tresser la métaphore de son roman L'interférence ou bien, pour emprunter les termes de l'analyse qu'établira Frantz Fanon à l'aune des guerres d'indépendance, «l'émaciation du panorama culturel». Après s'être jeté "frénétiquement dans l'acquisition forcenée de la culture de l'occupant ${ }^{54}$, Rabearivelo en arrive à un seuil critique : «l'esprit de ses aïeux » se présente voué à se réinventer dans les cadres impartis par cette Europe conquérante et hypocrite ${ }^{55}$ à laquelle, cependant, il aimerait laisser son nom comme un immortel sarcasme.

\section{Conclusion : dandysme et indigénat}

Rabearivelo, comme on a pu s'en apercevoir, oscille entre des vues assimilationnistes (militant auprès de l'Administration pour une "vie culturelle ») et des critiques parfois acerbes à l'encontre de la mission civilisatrice (à propos des guerres coloniales, du code de l'indigénat). Pour éclairer cette ambivalence, il faudrait mettre l'accent sur son esprit éminemment subversif. À tous les niveaux: littéraire (prouesses métriques, invention d'une écriture bilangue ${ }^{56}$ ), morale (libertinage physique et intellectuel, dignité du suicide ${ }^{57}$ ), nationaliste (monarchisme prononcé, allégeance à l'Action française)... Rabearivelo cultive un goût de la controverse, pourvu qu'elle soit constructive et participe à l'émergence d'une vie culturelle. C'est la marque du dandy de n'être d'aucun bord, sinon celui de l'Intelligence et de l'ego. Tel est le sens de ce 
néologisme : se mallarmiser, qui constitue une réponse au code de l'Indigénat. Pour un indigène brimé dans ses aspirations, le culte du moi (d'où Barrès) et la recherche de plaisirs supérieurs (tel Baudelaire) donnent sens à sa vie, sous l'œil des barbares ${ }^{58}$.

La nouvelle Le Drame de la maison Margon cultive par le biais de la fable cet art dandy du contrepoint, qui joue du contraste qu'offre « la feinte de luxe» du couple Margon avec l'élégance, à la fois dans les manières et par son esprit alerte, de leur employé de maison, Barthélémy Randevo. La fable participe de cette réponse de l'éthique dandy au code de l'indigénat. À ce propos, il est tout à fait significatif que le sixième tome des Calepins bleus - les cinq précédents ayant été brûlés par l'auteur ${ }^{59}$ - s'ouvre sur le nom scindé de Baudelaire : «Baude » est inscrit sur le cahier précédent, « laire» sur celui que nous pouvons lire aujourd'hui. À ce petit détail pas forcément dû au seul hasard s'ajoute la récurrence du nom de ce poète auquel Rabearivelo voue une admiration effrénée, allant jusqu'à se réciter les petits poèmes en prose ${ }^{60}$ en guise de prières. La littérature fait office de religion: la lecture étant pour Rabearivelo un acte de recueillement ${ }^{61}$; les manuscrits et les autographes, des reliques ou des fétiches; quant à l'écriture, elle est une illumination qu'il met en scène et cultive dans ses Calepins bleus.

Comment comprendre autrement l'ambivalence de Rabearivelo à l'égard de la V.V.S. ? Dénonçant l'arbitraire des chefs d'accusation - discours séditieux « anti-français » dans la presse - et appelant à une révision du procès pour laver et l'honneur des inculpés et la réputation de la justice française, Rabearivelo traite cette affaire de "ridicule". Évoquant la V.V.S., Rabearivelo ne peut s'empêcher de marquer son dédain. Cette attitude, qualifiée parfois de réactionnaire, provient davantage de son esprit libertin que de son collaborationnisme avec l'Administration. La divergence se situe sur le terrain religieux, d'abord et avant tout. Ridicule était à ses yeux l'ethos de ces pasteurs envers lesquels il fut, tout au long de sa vie, en délicatesse. Serge Meitinger met en avant sa relation conflictuelle avec le pasteur Ravelojaona, son oncle paternel. Mais c'est bien en raison d'une " hygiène morale " (fidélité à Baudelaire) qu'il regarde d'un mauvais œil ce mouvement, tout patriotique soit-il. Trop heureux cependant de soutenir un sursaut d'orgueil national dont il déplore l'absence chez un grand nombre de ses compatriotes ${ }^{62}$ - obnubilés par la seule naturalisation - il en prend la défense. La preuve en est qu'il s'engage dans une tribune politique; puis, à travers une nouvelle dans laquelle il a soin de souligner, justement, la «francophilie» de son héros et, surtout, sa vivacité d'esprit, son mérite. Le Drame de la maison Margon est un hymne à la méritocratie.

Le Drame de la maison Margon correspond au drame de Rabearivelo lui-même. La chute de la nouvelle est brutale et ne résout rien des frustrations essuyées par Randevo. La décadence funeste de la maison Margon renvoie au lecteur, sous un voile libertin, l'image d'un couple de colons sur la pente de la décivilisation ${ }^{63}$ - au point que la femme Gaby se suicide et le mari Louis trouve la mort au volant de sa voiture. Ne reste donc que ce magnifique Barthélémy Randevo, ou, plutôt, la "pelote de son aventure » et ses «drames » parmi lesquels celui de la maison Margon. Mais que devient Randevo, finit-il par trouver un poste en adéquation avec ses compétences? Nous savons seulement que c'est la peur d'un déclassement social (doublé d'un échec littéraire) qui précipita la mort du premier écrivain malgache d'expression française. Le personnage de Randevo ne cessa jamais de hanter Rabearivelo ; il en fut l'ombre prophétique. 


\section{BIBLIOGRAPHIE}

\section{Fuvres de référence}

RABEARIVELO, Jean-Joseph, CEuvres complètes. Tome $2:$ Le poète, le narrateur, le dramaturge, le critique, le passeur de langues, l'historien, Présence africaine, CNRS, coll. Planète libre, 2012.

RABEARIVELO, Jean-Joseph, CEuvres complètes. Tome 1 : Jean-Joseph Rabearivelo par lui-même : le diariste, "Les calepins bleus », l'épistolier, le moraliste, Présence africaine, CNRS, coll. Planète libre, 2010.

Jean-Joseph Rabearivelo, « Espace Afrique-Caraïbe », projet e-Man, Claire Riffard, équipe francophone, Institut des textes et manuscrits modernes, CNRS-ENS, [en ligne : http://emanarchives.org/francophone].

\section{Bibliographie générale}

BARRÈs, Maurice, « Réponse au discours de réception de Jean Richepin », discours prononcé à l'Académie française le 18 février 1909, Académie française [en ligne : http://www.academiefrancaise.fr/reponse-au-discours-de-reception-de-jean-richepin].

BARRÈs, Maurice, trilogie du Culte du moi : Sous l'œil des barbares; Un homme libre ; Le Jardin de Bérénice, Plon, 1922.

BERNARDIN DE SAINT-PIERRE, Henri, illustration de Roza, Gaude, Paul et Virginie, E. Figuière, 1924.

BOUDRY, Robert, préface de AMROUCHE, Jean, Jean-Joseph Rabearivelo et la mort, Présence africaine, 1958.

BowD, Gavin Philip, « Jean-Joseph Rabearivelo, Charles Maurras and colonial Madagascar », Modern \& Contemporary France, 24:1, 2016.

BUSTARRET, Claire, " Quand l'écriture vive devient patrimoine : Les manuscrits d'écrivains à l'Exposition de 1937 », Culture \& Musées, n 1, vol. 16, 2010, p. 159-176.

CÉSAIRE, Aimé, « L'homme de culture et ses responsabilités », Présence africaine, n² 24, 1959. COURTIN, Nicolas, « Du gouvernement royal des Hauts plateaux à l'État colonial français. L 'émergence de dispositifs de polices à Madagascar ", Crime, Histoire \& Sociétés / Crime, History \& Societies, $\mathrm{n}^{\circ} 2$, vol. 15, 2011, p. 77-95.

ESOAVELOMANDROSO, Manassé, " Une arme de domination : le "tribalisme" à Madagascar ", dans CHRÉTIEN, Jean-Pierre et PRUNIER, Gérard, Les ethnies ont une histoire, Paris, Karthala, coll. Hommes et sociétés, 2003.

JAEGLÉ, Eugène, Essai de bibliographie de Madagascar et dépendances, Tananarive, Bulletin économique de Madagascar, 1927.

LARBAUD, Valery, Ce vice impuni, la lecture, Paris, Gallimard, coll. Domaine anglais, 1936. LouP, Douna, L'Oragé, Mercure de France, 2015.

MALELA, Buata Bundu, Les écrivains afro-antillais à Paris. 1920-1960. Stratégies et postures identitaires, Paris, Karthala, coll. Lettres du sud, 2008. 
MATSUNUMA, Miho, « La colonisation européenne vue du Japon. Le cas de Yanaihara Tadao (1893-1961), principal acteur des études coloniales de l'entre-deux-guerres », dans BERTRAND, Romain, BLAIS, Hélène, SIBEUD, Emmanuelle (dir.), Cultures d'empires. Échanges et affrontements culturels en situation coloniale, Paris, Karthala, 2015.

MEITINGER, Serge, «Jean-Joseph Rabearivelo, poète de l'enracinement », Littérature, revue trimestrielle, $n^{\circ} 83,1991$.

MEITINGER, Serge, « Poésie malgache : Jean-Joseph Rabearivelo, Flavien Ranaivo et Jacques Rabemananjara », lrdb.fr, [en ligne : http://www.lrdb.fr/articles.php?lng=fr\&pg=384], 2007.

MEITINGER, Serge, « L'amour La poétique », Genesis, n 33, 2011.

MEITINGER, Serge, RIFFARD, Claire, DE BIASI, Pierre-Marc, CERQUIGLINI, Bernard, RAMAROSOA, Liliane, L'œuvre littéraire de Rabearivelo, "Prince des poètes malgaches », conférence enregistrée au salon de lecture Jacques-Kerchache le 21 septembre 2012, musée du quai Branly, 2012.

MONGO-MBOUSSA, Boniface, « René Maran, Léopold Sédar Senghor : une relecture ", Présence africaine, $\mathrm{n}^{\circ} 187-188,2013$.

MONTAGNÉ, Lucien, Essai de grammaire malgache, Société d'éditions géographiques, maritimes et coloniales, 1931.

OUATTARA, Bourahima, « Senghor, lecteur de Barrès ", Présence africaine, nº 191, 2015.

Rabemananjara, Jacques, « Le Poète noir et son Peuple », Présence africaine, n 16, 1975.

RABEMANANJARA, Jacques, « $50^{\mathrm{e}}$ anniversaire de la mort de Jean-Joseph Rabearivelo », Présence africaine, $\mathrm{n}^{\circ} 142,1987$.

RABEMANANJARA, Jacques, entretien avec COMPAN, Magali, Présence africaine, nº 171, 2005.

RAISON-JOURDE, Françoise (dir.), Les Souverains de Madagascar. L'histoire royale et ses résurgences contemporaines, Karthala, coll. Hommes et sociétés, 1983.

RAMAROSOA, Liliane, Itinéraires de la littérature malgache d'expression française de 1923 à 1980. Essai d'histoire littéraire, thèse d'État, Paris 4-Sorbonne, 1992.

RAVALITERA, Pela, « Madagascar : l'Académie malgache, dans le programme de colonisation de Gallieni », AllAfrica.com,15/7/2015, consulté le 12/2/2017, http://fr.allafrica.com/stories/ 201507171000.html.

RAZAFINTSAMBAINA, Gabriel, « Hommage à Rabearivelo... », Présence africaine, n 36, 1961.

RENAULT, Mathieu, « Amour de la race ou amour au-delà des races ? Frantz Fanon, lecteur de René Maran », Présence africaine, n 187-188, 2013, p. 231-244.

RENEL, Charles, Le Décivilisé, Flammarion, 1923.

RIFFARD, Claire, «Écrire en deux langues », Item.ens.fr, mis en ligne le 7/7/2008, consulté le 10/2/2017 http://www.item.ens.fr/index.php?id=344663.

RIFFARD, Claire, «J.-J. Rabearivelo, Prince des poètes malgaches ", Cultures Sud, n 164, janvier 2007, p. 110-123.

RIFFARD, Claire, Mouvements d'une écriture. La poésie « bilangue » de Presque-Songes et Traduit de la nuit de Jean-Joseph Rabearivelo, thèse de doctorat soutenue à l'université Paris 13-Villetaneuse, 2006.

SEGALEN, Victor, Les Immémoriaux, Mercure de France, 1907. 
SENGHOR, Léopold Sédar, Anthologie de la nouvelle poésie nègre et malgache de langue française, PUF, 1948.

SEILER-DIETRICH, Almut « Comptes rendus de lecture », Présence africaine, n 184, 2011.

PIAZZA, Pierre, Aux origines de la police scientifique. Alphonse Bertillon, précurseur de la science du crime, Paris, Karthala, coll. Hommes et sociétés, 2011.

RANDRIANJA, Solofo, Société et luttes anticoloniales à Madagascar de 1896 à 1946, Karthala, coll. Hommes et sociétés, 2001.

Royal United Service Institution, The Madagascar expedition of 1895-96, summary from the official report of General Duchesne dated 25th April 1896, p. 41.

STEINS, Martin, Les Antécédents et la genèse de la négritude senghorienne, Paris-III, 1981.

VAILLANT, Janet (traduit de l'anglais américain par Jacques Meunier), Vie de Léopold Sédar Senghor. Noir, Français et Africain, Paris, Karthala, 2006.

VALERY, Paul, La crise de l'esprit, dans Euvres I, Gallimard, NRF, bibliothèque de la Pléiade, 1957.

VALERY, Paul, Regards sur le monde actuel, dans CEuvres II, Gallimard, NRF, bibliothèque de la Pléiade, 1960.

\section{NOTES}

1. LARBAUD, Valery, Ce vice impuni, la lecture, Gallimard, coll. Domaine anglais, 1936, p. 11.

2. RABEARIVELO, Jean-Joseph, CEuvres complètes, tome 1, Serge Meitinger, Liliane Ramarosoa, Claire Riffard (éd.), Paris, CNRS Éditions, coll. Planète libre, 2010.

3. el mouhoub AmRouche, Jean, yacine-TitouH, Tassadit (éd.), Journal (1928-1962), Paris, Non Lieu, 2009.

4. SEILER-DIETRICH, Almut, « Comptes rendus de lecture », Présence africaine, n 184, 2011.

5. «Gonzague Raynaud note dans son introduction au recueil Traduit de la nuit, Paris, 1990, p. $7:$ : Son journal [...] n'est encore ni traduit ni édité », dans SEILER-DIETRICH, Almut, « Comptes rendus de lecture », Présence africaine, $\mathrm{n}^{\circ}$ 184, 2011.

6. RIFFARD Claire, Mouvements d'une écriture. La poésie «bilangue » de Presque-Songes et Traduit de la nuit, de Jean-Joseph Rabearivelo, thèse de doctorat soutenue à l'université Paris 13, 2006.

7. Ces deux textes d'hygiène morale correspondent à la partie «Le moraliste ». Dans Le Bijou rose et noir, il y cultive un goût pour le "paradoxe moral autant qu'esthétique » développant une "conception aristocratique et dédaigneuse, prompte à railler le vulgaire ou les vaines prétentions comme à s'en distinguer» (tome I, p. 1166). D'un belluaire est de la même veine, quoique le "caractère lapidaire ou laconique » lui confère une sorte de "nudité désespérée " (tome I, p. 1187) bien particulière.

8. RABEARIVElo, Jean-Joseph, CEuvres complètes, tome 2, MEITINGER, Serge, INK, Laurence, RAMAROSOA, Liliane, RIFFARD, Claire (éd.), Paris, CNRS Éditions, coll. Planète libre, 2012, 1792 p.

9. http://eman-archives.org/francophone/collections/show/2

10. BUSTARRET, Claire, «Quand l'écriture vive devient patrimoine : les manuscrits d'écrivains à l'Exposition de 1937 », Culture et musées, n 1, vol. 16, 2010, p. 159-176.

11. Calepins bleus, tome I, op. cit., 3/8/1935, p. 883.

12. Rabearivelo le nomme de manière laconique «l'auteur de l'Essai». Il lui sait gré d'avoir « mis en évidence sur la carte de l'Esprit [...] le riche royaume mélanien ». Ne serait-ce que pour cette $d$ écouverte anthropologique, il lui «serait déjà bien cher " souligne-t-il dans ses Calepins le 15 mai 
1934 à la suite d'une mauvaise critique parue dans les colonnes de l'hebdomadaire parisien Vendé miaire. Gobineau, cet audacieux penseur de l'aryanisme, quoique excentrique - par ses envolées métaphysiques et le ton péremptoire avec lequel il soutient sa thèse explicative de la chute des civilisations sous le seul prisme de la dégénérescence liée au métissage - était au cœur de l'intelligentsia européenne du XIX siècle. Entretenant une "relation réglée " avec Ernest Renan (1823-1892), une correspondance épistolaire avec Alexis de Tocqueville (1805-1859), dont il était le chef de cabinet aux Affaires étrangères, cela, dans le sillage du Premier ministre britannique Benjamin Disraeli (1804-1881), il se situe à la confluence des courants libéraux qui inondent la bibliothèque coloniale.

13. [Votre questionnaire me laisse bien perplexe], [en ligne: http://eman-archives.org/ francophone/items/show/1931], 26/3/1935.

14. Calepins bleus, tome I, op. cit., 8/7/1934, p. 564-565.

15. BARRÈs, Maurice, «Réponse au discours de réception de Jean Richepin », discours prononcé à l'Académie française le 18 février 1909, Académie française [en ligne: http://www.academiefrancaise.fr/reponse-au-discours-de-reception-de-jean-richepin].

16. Calepins bleus, tome I, op. cit., 8/7/1934, p. 560.

17. Loin d'être incongrue, l'influence barrésienne s'exerce chez Senghor ; voir STEINS, Martin, Les Antécédents et la genèse de la négritude senghorienne, Paris 3, 1981.

18. Calepins bleus, tome I, op. cit., 15/1/1934, p. 344.

19. ESOAVELOMANDROSo, Manassé, "Une arme de domination : le "tribalisme" à Madagascar», CHRÉTIEN, Jean-Pierre et PRUNIER, Gérard, Les ethnies ont une histoire, Paris, Karthala, coll. Hommes et sociétés, 2003.

20. MONTAGNÉ, Lucien, Essai de grammaire malgache, Société d'éditions géographiques, maritimes et coloniales, 1931, p. 6.

21. PRO MAN1 MAISON MORGON 2.jpg, [en ligne : http://eman-archives.org/francophone/files/ show/3916].

22. PIAZZA, Pierre, Aux origines de la police scientifique. Alphonse Bertillon, précurseur de la science du crime, Paris, Karthala, coll. Hommes et sociétés, 2011, p. 276.

23. «Au pays de Lombroso", [en ligne: http://eman-archives.org/francophone/items/show/ 2196], 12/2/1935.

24. VINCHON, Jean, L'Art et la Folie, Paris, Stock, 1924.

25. Calepins bleus, tome I, op. cit., 11/2/1936, p. 1002.

26. ESOAVELOMANDROSO, Manassé, « Une arme de domination : le "tribalisme” à Madagascar ", dans CHRÉTIEN, Jean-Pierre, PRUNIER, Gérard, Les ethnies ont une histoire, Paris, Karthala, coll. Hommes et sociétés, 2003, p. 259.

27. GLISSANT, Édouard, Le Discours antillais, Seuil, 1981, p. 194.

28. Le Drame de la maison Margon, PRO MAN1 MAISON MORGON 2.jpg, [en ligne : http://emanarchives.org/francophone/files/show/3915].

29. MEITINGER, Serge, «Introduction générale », tome 1, op. cit., p. 24.

30. Ce trait d'humour, quoique teinté d'ironie désabusée, a été érigé en épitaphe littéraire par Senghor au sein de la notice de présentation qu'il rédige pour chacun des poètes de l'Anthologie de la nouvelle poésie nègre et malgache de langue française: « S'il comprenait assez bien le français à sa sortie de l'école, il l'écrivait mal. C'est alors que l'enfant malgache, à peine adolescent, accomplit ce prodige, qui restera dans l'histoire des Lettres, de faire du français, au bout de quelques années de labeur acharné, sinon méthodique, un instrument docile à son génie. Il fit mieux, il s'assimila le génie français avec la langue au point d'être véritablement, comme il l'écrit quelques heures avant sa mort, «naturellement Latin chez les Mélaniens. Et avec les traits de ceux-ci », op. cit., p. 179. 
31. Expliquant les raisons l'ayant conduit à entrer au Cercle de Tananarive comme bibliothécaire, le narrateur confie à son compagnon qu'il «ne [connaissait] les Européens qu'en des rapports vraiment extérieurs ». Ainsi, « après mûre réflexion », il se dit : « Pourquoi ne souffrais-tu pas un long séjour ainsi désavantageux pour toi, sinon pour connaître à fond ton sujet d'étude, du moins pour le pénétrer? ». Ce à quoi il s'est « résigné » (voir PRO MAN1 MAISON MORGON 11.jpg, [en ligne : http://eman-archives.org/francophone/files/show/3924]).

32. Dans ses Regards sur le monde actuel, Paul Valéry propose d' « imaginer le pire » afin de prendre la mesure de la «décadence de l'Europe » que la Grande Guerre «n'a fait qu'accuser et précipiter » : «Considérez un peu ce qu'il adviendra de l'Europe quand il existera par ses soins, en Asie, deux douzaines de Creusot ou d'Essen, de Manchester, ou de Roubaix, quand l'acier, la soie, le papier, les produits chimiques, les étoffes, la céramique et le reste y seront produits en quantité écrasantes, à des prix invincibles, par une population qui est la plus sobre et la plus nombreuse du monde, favorisée dans son accroissement par l'introduction des pratiques de l'hygiène » (Euvres II, Gallimard, Pléiade, 1960, p. 927).

33. Nouvelle Revue française, $\mathrm{n}^{\circ} 71,1^{\mathrm{er}}$ août 1919 ; Variété, Gallimard, 1924 ; Variété I, Gallimard, 1934.

34. Stock, 1931 ; Flammarion, 1933.

35. Calepins bleus, tome I, op. cit. (16/9/1933, p. 218) ; « Le dentiste Rajoelina [...] sa façon de saluer à la bourjane, à l'esclave» $(18 / 1 / 1934$, p. 350$)$; "Vous descendriez peut-être aussi de ces «Messieurs » et non des esclaves que nous avions échangés contre des fusils à la Réunion?» $(16 / 2 / 1934$, p. 379$)$; « mon Jean-Baptiste [...] outré de savoir que nous sommes assez nombreux ici qui adhérons au monarchisme, cet esclave parvenu n'eut de cesse d'en faire part à l'Autorité Supérieure » (29/5/1934, p. 517) ; « le prince Ramaka, jadis, au temps malgache, avait cinq belles esclaves » qu'il pouvait « culbuter » à souhait : « N'est-ce pas merveilleux! » (10/9/1935, p. 899); «Une femme épilée, à quelque race qu'elle appartienne, ce n'est qu'une esclave. Telle a été, [...] hier, mon impression en faisant le Fils auprès de ma petite animale.» (7/2/1936, p. 1000). Ses deux romans portent la nostalgie de la royauté du siècle passée durant laquelle les propriétaires fonciers disposaient librement d'une force de travail et d'un harem. L'économie du royaume était insérée dans une économie de traite (européenne et arabo-musulmane), de sorte que ceux-ci pouvaient, à tout moment, s'ils avaient à se plaindre d'un ou d'une de leurs esclaves, le/la « vendre à la côte » (L'Interférence, tome 2, op. cit., p. 995).

36. CÉSAIRE, Aimé, Discours sur le colonialisme, Paris, Présence africaine, 1955 (1950).

37. Évoquant un projet de revue : « FINE CIGARETTE, courrier de littérature et d'art de J.J. R. Parution irrégulière : deux à trois fois par an. Huit pages ", il pense à l'usage qu'il en ferait : "Je m'en servirai pour donner mon opinion sur les Livres et les Hommes. » Il poursuit en imaginant une vie d'écrivain idéale, serviteur de la seule Intelligence : « Être chez moi et pouvoir penser et agir dans ce sens. N'être plus à charge, n'avoir plus à passer tel écrit sous les yeux et selon le bon plaisir de tel Béotien que je méprise mais qui se trouve être, dans la vie, mon supérieur ", Calepins bleus, 8/5/1933, tome I, op. cit., p. 93.

38. [Votre questionnaire me laisse bien perplexe], [en ligne : NUM ETU MAN1 Questionnaire 7]. 39. À propos de René Maran, voir à ce sujet : VAILlANT, Janet (traduit de l'anglais américain par Jacques Meunier), Vie de Léopold Sédar Senghor. Noir, Français et Africain, Paris, Karthala, 2006. Biographie sur laquelle s'appuie Boniface Mongo-Mboussa pour considérer l'intention de René Maran : " "Dans son plaidoyer en faveur d'une réforme coloniale, Maran ne parlait pas en tant que membre d'un groupe colonisé ni même de son point de vue propre, mais comme un Français pressant d'autres Français à mettre de l'ordre dans leur maison commune." Certes, René Maran a été à cause de sa préface vénéneuse de Batouala, victime d'un ostracisme littéraire et politique. Il a été ensuite, par la force des événements, contraint à s'approcher des "mouvements nègres" à Paris pour reprendre le titre de l'essai de Philippe Dewitte. Au fond, il appartenait à cette génération d'Antillais décrite par Maryse Condé dans son Le cœur à rire et à pleurer et stigmatisée 
par Césaire dans Nègreries» (MONGO-MBOUSSA, Boniface, «René Maran, Léopold Sédar Senghor : une relecture ", Présence africaine, $\mathrm{n}^{\circ}$ 187-188, 2013).

40. Ce capital symbolique que revêt la langue française a déjà fait l'objet de nombreuses études. La présentation qu'en a faite Serge Meitinger, que ce soit dans les deux tomes des éditions scientifiques ou au travers de ses articles de revues, est suffisamment étayée pour que l'on s'y attarde à nouveau. En outre, c'est bien cette grille de lecture qui a toujours prévalu : comment Rabearivelo fut épris de la langue française au point d'en être ébloui, parvenant difficilement à se défaire de ses "réminiscences", concluaient les analyses les plus radicales; quant aux autres, elles s'attachèrent à souligner la «bestialité » de son apprentissage et de sa culture littéraire, jusqu'à faire de sa vie une odyssée spirituelle. L'intérêt d'une étude sur cette fascination qu'exerce la Francophonie et, plus généralement, l'Occident (imaginé ou réel) serait davantage du côté de sa réception à Madagascar, des années 1930 à aujourd'hui.

41. matsunuma, Miho, «La colonisation européenne vue du Japon. Le cas de Yanaihara Tadao (1893-1961), principal acteur des études coloniales de l'entre-deux-guerres », dans BERTRAND, Romain, BLAIS, Hélène, SIBEUD, Emmanuelle (dir.), Cultures d'empires. Échanges et affrontements culturels en situation coloniale, Paris, Karthala, 2015.

42. PRO MAN1 MAISON MORGON 1.jpg, [en ligne : http://eman-archives.org/francophone/files/ show/3915].

43. NUM ETU MAN1 Procès, http://eman-archives.org/francophone/admin/files/show/3411

44. [Non une amnistie, mais une révision !], [en ligne : http://eman-archives.org/francophone/ items/show/1933].

45. « Aux Muses », tome 2, op. cit., p. 414-415.

46. [Non une amnistie, mais une révision !], [en ligne : http://eman-archives.org/francophone/ items/show/1933].

47. Calepins bleus, tome I, op. cit., 6/5/1933, p. 91.

48. Calepins bleus, tome I, op. cit., 31/5/1933, p. 111.

49. Ny Fandrosoam-baovao, Nouvelle série, $2^{\mathrm{e}}$ année, $\mathrm{n}^{\circ}$ 41, Tananarive, 22 juin 1932 ; tome 2, op. cit., p. 1299.

50. "À Philippe Chabaneix », Volumes, op. cit., p. 263. Tel est le motif de sa "rêverie historique » dans Tananarive, le triptyque de sa poésie : retrouver, dans l'orientation des maisons, la végétation, etc., ce qui fait la marque des « vieilles races », MS1.POTA, [en ligne : http://eman-archives.org/ francophone/items/show/1814].

51. SEGALEN, Victor, Les Immémoriaux, Mercure de France, 1907.

52. D'un point de vue génétique, témoignent de cette hantise les inscriptions au recto du manuscrit de sa réponse au questionnaire sur la jeunesse noire: "Immémoriaux» et «Océaniens », « Impersonnels » : « sans traditions », « sans passé ». En témoigne aussi ce projet de roman : «Je veux parler de mes projets. / Il y a surtout un grand roman, L'Âme des Morts, dont les deux premières parties, entièrement "pensées" déjà, sont prêtes à être écrites. Mais j'attends des jours plus clairs. / Ces deux parties - "livres" - auront respectivement pour titre : Les Hommes et les Choses et Les Morts et les Vivants. / [...] je suis [...] en train d'en créer, d'en établir, l'atmosphère, l'ambiance. Tout tiendra, je crois, dans ces premières phrases provisoires qui ouvriront l'ouvrage : / “Calmes et froids jusqu'à la désolation, ces grands paysages de collines font l'Imerina. Ils s'étendent à perte de vue et se confondent avec la ligne bleue de l'horizon. Des nomades de l'océan, venus d'on ne sait exactement plus où, les avaient découverts et élus de temps immémorial. Leurs tombeaux..." / Puis, nouant et prolongeant les mailles du temps, les Vivants seront évoqués. / Tout y sera donc expliqué aussi..." (Calepins bleus, tome I, op. cit., 5/1/1934, p. 319-321). Ailleurs encore, à propos d'une rumeur de francisation des noms malgaches, Rabearivelo est assailli par ce spectre : "Ce serait tout simplement ridicule qu'un Rakoto devint un Racoute ou un Racouteau ! / Hein! Mais qu'est-ce que je me disais naguère : bientôt, nous, vous, eux... / Tous ces "Impersonnels"! Ces pauvres, ces lamentables, 
"Impersonnels" qui seront également, avant longtemps, des "Immémoriaux" " (Calepins bleus, tome I, op. cit., 22/3/1935, p. 800). On le voit, Rabearivelo est hanté par ce que l'ethnologue Robert Jaulin appellera la « paix blanche ».

53. Volumes, tome II, op. cit., p. 264.

54. FANON, Frantz, Les damnés de la terre, La Découverte, Paris, [1961], 2002, p. 226.

55. BowD, Gavin Philip, « Jean-Joseph Rabearivelo, Charles Maurras and colonial Madagascar ", Modern \& Contemporary France, 24:1, 2016.

56. RIFFARD, Claire, Mouvements d'une écriture. La poésie «bilangue» de Presque-Songes et Traduit de la nuit, de Jean-Joseph Rabearivelo, thèse de doctorat soutenue à l'université Paris 13-Villetaneuse, 2006 ; «Écrire en deux langues », Item.ens.fr, mis en ligne le 7/7/2008, consulté le 10/2/2017, [en ligne : http://www.item.ens.fr/index.php?id=344663].

57. Calepins bleus, tome I, op. cit., 20/2/1936, p. 1005-1006.

58. BARRÈs, Maurice, trilogie du Culte du moi : Sous l'œil des barbares; Un homme libre; Le Jardin de Bérénice, Plon, 1922.

59. «Celui-ci échappera-t-il à ce sort cendreux? » s'interroge-t-il dans ses Calepins bleus en date du 5 janvier 1933 (tome I, op. cit., p. 85).

60. Voir Calepins bleus du 3/2/1934 (tome I, op. cit., p. 361) et du 13/10/1934 (tome I, op. cit., p. 631).

61. Voir le poème d'ouverture du recueil bilingue Sari-nofy/Presque-songes, " Mamaky teny/Lire », tome 2, p. 514-515.

62. Colonial \& Malagasy, 10/10/1934.

63. RENEL, Charles, Le Décivilisé, Flammarion, 1923.

\section{RÉSUMÉS}

Cet article propose de lire le manuscrit d'une nouvelle inédite de Jean-Joseph Rabearivelo comme une fable de l'Histoire dans laquelle se condenserait, d'une part, une veine ethno-nationale marquée par l'état centralisateur précolonial (le royaume de l'Imerina) irriguée par les mouvements régionalistes français; et, d'autre part, une conscience d'intellectuel colonisé qui emprunte autant aux théories raciales des années folles qu'elle articule un discours critique sur la présence française à Madagascar.

\section{INDEX}

Mots-clés : Rabearivelo, Madagascar, manuscrit, génétique, Valery Larbaud, René Maran, Paul Valéry

\section{AUTEUR}

\section{XAVIER LUCE}

Doctorant, université Paris 4-Sorbonne 